\title{
Quantum correlations are not contained in the initial state*
}

\author{
Adán Cabello ${ }^{\dagger}$ \\ Departamento de Física Aplicada, Universidad de Sevilla, 41012 Sevilla, Spain
}

(May 26, 2022)

\begin{abstract}
Two proofs are presented which show that quantum mechanics is incompatible with the following assumption: all possible correlations between subsystems of an individual isolated composite quantum system are contained in the initial quantum state of the whole system, although just a subset of them is revealed by the actual experiment.
\end{abstract}

PACS number(s): 03.65.Bz

\section{INTRODUCTION}

Since the seminal work of Einstein, Podolsky, and Rosen [1], the entanglement between quantum variables pertaining two different parts of a composite system has been considered the characteristic trait of quantum mechanics 22]. Recently discovered phenomena involving composite systems of more than two parts, such as quantum dense coding [3, [4, teleportation of quantum states [5], and entanglement swapping [5,8 10], have in common that they exploit the fact that in a multiparticle system, entanglement between two parts can be considered itself an entangled property [11]. The implications of these phenomena to several proposed interpretations of quantum mechanics are currently subject of investigation 12. In this context, I have argued recently that a certain interpretation of quantum mechanics based on the assumption that correlations between subsystems of an individual isolated composite quantum system are real objective local properties of that system 13 15 is inconsistent 16]. My argument was based on an example: consider two pairs of spin- $\frac{1}{2}$ particles prepared so that both pairs are in the singlet state (the first pair is composed by particles 1 and 2, and the second by particles 3 and 4). By performing one among two alternative measurements on particles 2 and 3, one can choose between two types of correlations for particles 1 and 4 (spacelike separated from the measurement on particles 2 and 3): they can be in a pure factorizable state or, alternatively, in a pure maximally entangled state [16. This then would allow one to choose nonlocally the type of correlations between two distant parts. In my opinion, this is inconsistent with the assumption that such correlations were local objective properties. However, in [16] I wrote 'I do

*Accepted for publication in Phys. Rev. A.

†Electronic address: fite1z1@sis.ucm.es not mean that the internal correlations between particles 1 and 4 "change" after a spacelike separated experiment (this does not happen in the sense that no new internal correlations are "created" that were not "present" in the reduced density matrix for the system 1 and 4 before any interaction), but that the type of internal correlations (and therefore, ... the reality) of an individual isolated system can be chosen at distance.' So, implicitly, I admitted that all such possible correlations between two parts were present somehow in the initial quantum state of the whole system, although just a subset of them is revealed by the actual experiment. The aim of this note is to show that even such an innocuous-looking assumption is incompatible with quantum mechanics. For that purpose I will present two proofs in which such an assumption leads to a contradiction. The first is a GreenbergerHorne-Zeilinger-like proof 17 19] involving three pairs of spin- $\frac{1}{2}$ particles. This proof does not require inequalities nor probabilities, indeed it also admits a reading as a multiplicative proof of the Kochen-Specker theorem [20,21 in a Hilbert space of dimension sixty four. The second proof is even simpler. It is a Hardy-like proof without inequalities (but with probabilities) [22] involving two pairs of spin- $\frac{1}{2}$ particles.

\section{GHZ-LIKE PROOF OF IMPOSSIBILITY OF PREEXISTENT CORRELATIONS}

For the first proof consider three pairs of spin- $\frac{1}{2}$ particles labeled from 1 to 6 . The Hilbert space in which we describe the spin state of this system has dimension sixty four. I will call it $\mathcal{H}_{64}$. Let $A_{i j}$ be the non-degenerate operator acting on the four-dimensional subspace of particles $i$ and $j$, defined as

$$
A_{i j}=2 \hat{\alpha}_{i j}^{++}+\hat{\alpha}_{i j}^{+-}-\hat{\alpha}_{i j}^{-+}-2 \hat{\alpha}_{i j}^{--},
$$

where $\hat{\alpha}_{i j}^{+-}$is the projection operator onto the state $\left|\alpha^{+-}\right\rangle_{i j}=|+\rangle_{i} \otimes|-\rangle_{j}$, etc. Let $B_{i j}$ be the non-degenerate Bell operator 23] defined as

$$
B_{i j}=2 \hat{\phi}_{i j}^{+}+\hat{\psi}_{i j}^{+}-\hat{\psi}_{i j}^{-}-2 \hat{\phi}_{i j}^{-}
$$

where $\hat{\phi}_{i j}^{+}$is the projection operator onto the state $\left|\phi^{+}\right\rangle_{i j}$, etc., being

$$
\begin{aligned}
\left|\phi^{ \pm}\right\rangle_{i j} & =\frac{1}{\sqrt{2}}\left(|+\rangle_{i} \otimes|+\rangle_{j} \pm|-\rangle_{i} \otimes|-\rangle_{j}\right), \\
\left|\psi^{ \pm}\right\rangle_{i j} & =\frac{1}{\sqrt{2}}\left(|+\rangle_{i} \otimes|-\rangle_{j} \pm|-\rangle_{i} \otimes|+\rangle_{j}\right),
\end{aligned}
$$


the four Bell states [23], which form an orthogonal basis for the corresponding four-dimensional subspace.

Consider now the four operators acting on $\mathcal{H}_{64}$ defined as

$$
\begin{aligned}
& A_{12} A_{34} B_{56}=A_{12} \otimes A_{34} \otimes B_{56}, \\
& A_{12} B_{34} A_{56}=A_{12} \otimes B_{34} \otimes A_{56}, \\
& B_{12} A_{34} A_{56}=B_{12} \otimes A_{34} \otimes A_{56}, \\
& B_{12} B_{34} B_{56}=B_{12} \otimes B_{34} \otimes B_{56} .
\end{aligned}
$$

As it can be easily checked, any of these four operators has eigenvalues $\pm 2^{k}$, with $k=0,1,2,3$. In addition, the four operators are mutually commutative so they possess a set of common eigenvectors. Let one of these common eigenvectors be the initial state of the six-particle system; for instance, the state $|\mu\rangle$ defined by the following eigenvalue equations:

$$
\begin{aligned}
& A_{12} A_{34} B_{56}|\mu\rangle=|\mu\rangle, \\
& A_{12} B_{34} A_{56}|\mu\rangle=|\mu\rangle, \\
& B_{12} A_{34} A_{56}|\mu\rangle=|\mu\rangle, \\
& B_{12} B_{34} B_{56}|\mu\rangle=-|\mu\rangle .
\end{aligned}
$$

Note that the four respective eigenvalues $(1,1,1$, and -1 , in this case) are not independent since they must obey the same functional relations satisfied by the four operators. In particular, since the product of the four operators is a negative operator (i.e., all its eigenvalues are negative numbers) with eigenvalues $-16^{m}$, with $m=0,1,2,3$, then the product of their four eigenvalues must be negative.

Now let us assume, as it is in $13 \sqrt{15}$, that all the correlations between subsystems of the composed system are real objective internal local properties of such subsystems. In particular, consider three subsystems: the first is composed by particles 1 and 2 , the second by particles 3 and 4 , and the third by particles 5 and 6 . We will assume that all possible correlations between particles 1 and 2 (for instance) are encoded in the initial state for the whole system, and they do not depend on any interaction experienced by the other subsystems, so they cannot change (in particular, they cannot be created) as a result of any experiment performed on particles 3 to 6 (supposed to be spacelike separated from particles 1 and 2).

Now consider three observers, each having access to one pair of particles. On each pair, they may measure either $A_{i j}$ or $B_{i j}$, without disturbing the other pairs. The results of these measurements will be called $a_{i j}$ or $b_{i j}$, respectively. Since these results must satisfy the same functional relations satisfied by the corresponding operator, then, from (9), we can predict that, if $A_{12}, A_{34}$, and, $B_{56}$ are measured, the results satisfy

$$
a_{12} a_{34} b_{56}=1 .
$$

Analogously, from Eqs. (10)-(12), the results of other possible measurements satisfy

$$
\begin{aligned}
a_{12} b_{34} a_{56} & =1, \\
b_{12} a_{34} a_{56} & =1, \\
b_{12} b_{34} b_{56} & =-1 .
\end{aligned}
$$

We can associate each one of the eigenvalues $a_{i j}$ and $b_{i j}$ with a type of correlation between particles $i$ and $j$ initially hidden in the original state of the system, but "revealed" by performing measurements on the two other distant pairs. For example, if $B_{12}$ and $B_{34}$ are measured and their results are both 1 , then one can predict with certainty that particles 5 and 6 are in the singlet state, and since arriving to this conclusion does not require any real interaction on particles 5 and 6 , then we assume that the spins of particles 5 and 6 were initially correlated in the singlet state (i. e., the same spin component of particles 5 and 6 would have opposite signs), so we assign the value -1 for the observable $B_{56}$ to the initial state $|\mu\rangle$. Alternatively, since a different measurement on particles 1 to 4 (for instance, by measuring $A_{12}$ instead of $B_{12}$ ) allows one to predict with certainty, without interacting with particles 5 and 6 , how the $z$ spin components of particles 5 and 6 are correlated, and since this information do not require any real interaction on particles 5 and 6 , then we suppose that it was encoded somehow in the initial state of the whole system (so we assign to the initial state one of the eigenvalues of $A_{56}$ ). Such predictions with certainty and without interaction would lead us to assign values to the six types of correlations given by $A_{12}, B_{12}, A_{34}, B_{34}, A_{56}$, and $B_{56}$. However, such an assignment cannot be consistent with the rules of quantum mechanics because the four equations (13)-(16) cannot be satisfied simultaneously, since the product of their left-hand sides is a positive number (because each value appears twice), while the product of the right-hand sides is -1 . Therefore, the whole information on the correlations between the particles of the three pairs cannot be encoded in the initial state as we assumed.

\section{PROOF OF THE KOCHEN-SPECKER THEOREM IN $\mathcal{H}_{64}$}

A similar argument could be developed starting from any common eigenvector of the four operators (5)-(8). In fact, including these four operators, the argument can be rearranged as a state-independent proof of the KochenSpecker theorem [20] (like the one proposed in [21]) in a sixty four dimensional Hilbert space. This proof is summarized in Fig. 1.

Fig. 1 contains ten operators: the four operators (5)(8) acting on the whole system, and six operators acting only on pairs of particles (two operators for each pair). The four operators on each of the five lines are mutually commutative. As told before, the product of the four operators on the horizontal line is a negative operator, and as can be easily verified, the product of the four operators on each of the other lines is one (and the same) positive 
operator (with eigenvalues $4^{n}$, with $n=0,1,2,3$ ). It is easily checked that it is impossible to ascribe one of their eigenvalues to the ten operators, satisfying all the same functional relations that are satisfied by the corresponding operators.

\section{HARDY-LIKE PROOF OF IMPOSSIBILITY OF PREEXISTENT CORRELATIONS}

The second argument against the possibility of predefined correlations is simpler. It requires just two pairs of spin- $\frac{1}{2}$ particles. Consider the following initial state:

$$
\begin{aligned}
|\eta\rangle= & \frac{1}{2 \sqrt{3}}(|+-+-\rangle-|+--+\rangle \\
& -|-++-\rangle-3|-+-+\rangle),
\end{aligned}
$$

where $|+-+-\rangle=|+\rangle_{1} \otimes|-\rangle_{2} \otimes|+\rangle_{3} \otimes|-\rangle_{4}$, etc. As it can be easily checked, this state has the following four properties:

$$
\begin{aligned}
P_{\eta}\left(\psi_{12}^{-} \mid \alpha_{34}^{+-}\right) & =1 \\
P_{\eta}\left(\psi_{34}^{-} \mid \alpha_{12}^{+-}\right) & =1 \\
P_{\eta}\left(\alpha_{12}^{+-}, \alpha_{34}^{+-}\right) & =\frac{1}{12} \\
P_{\eta}\left(\psi_{12}^{-}, \psi_{34}^{-}\right) & =0 .
\end{aligned}
$$

Property (18) tells us that on every copy of the system initially prepared in the state (17) in which the result of measuring $A_{34}$ is $\alpha_{34}^{+-}$, one can predict (with certainty and without interacting with them) that particles 1 and 2 are in the singlet state. Therefore, we conclude that in that subensemble of copies, particles 1 and 2 were initially in the singlet state. Analogously, property (19) tells us that on every copy of the system initially prepared in the state (17) in which the result of measuring $A_{12}$ is $\alpha_{12}^{+-}$, one can predict (with certainty and without interacting with them) that particles 3 and 4 are in the singlet state. Therefore, we conclude that in that subensemble, particles 3 and 4 were initially in the singlet state. Property (20) reveals that the intersection between the two subensembles defined before is not zero, since there is a non-zero probability to obtain simultaneously the two conditions defining such subensembles. Assuming that the predicted correlations were encoded in the initial state of the system, the previous properties would lead us to conclude that the probability of finding both pairs of particles in the singlet state is greater or equal than the probability of finding simultaneously the corresponding conditions, given in (20). However, property (21) shows that this is no so. In fact, the probability of finding two singlets is zero. Therefore, the assumption that these correlations were contained in the initial state is untenable.

\section{CONCLUSIONS}

"No-go" proofs show that in QM local observables cannot have predefined values $17,19,21,22$. In this paper I have shown how, by duplicating the number of involved particles, these proofs can be rearranged so as to exclude the possibility of predefined local correlations between two particles of a composite system. This impossibility would be taken into account in any attempt to describe phenomena such as quantum dense coding, teleportation of quantum states or entanglement swapping in a consistent interpretation of QM. In particular, this impossibility of preexistent correlations constraints any further development of the tentative interpretation proposed in [13 15.

\section{AKNOWLEDGMENTS}

The author thanks Gonzalo García de Polavieja for fruitful discussions, and Christopher Fuchs, David Mermin, and Asher Peres for useful feedback. Also, thanks are due to José Luis Cereceda for pointing out a mistake in a previous version. This work was financially supported by the Universidad de Sevilla (OGICYT-191-97) and the Junta de Andalucía (FQM-239).

[1] A. Einstein, B. Podolsky, and N. Rosen, Phys. Rev. 47, 777 (1935).

[2] E. Schrödinger, Proc. Cambridge Philos. Soc. 31, 555 (1935).

[3] C. H. Bennett and J. Wiesner, Phys. Rev. Lett. 69, 2881 (1992).

[4] K. Mattle, H. Weinfurter, P. G. Kwiat, and A. Zeilinger, Phys. Rev. Lett. 76, 4656 (1996).

[5] C. H. Bennett, G. Brassard, C. Crépeau, R. Jozsa, A. Peres, and W. K. Wootters, Phys. Rev. Lett. 70, 1895 (1993).

[6] D. Bouwmeester, J. Pan, K. Mattle, M. Eibl, H. Weinfurter, and A. Zeilinger, Nature 390, 575 (1997).

[7] D. Boschi, S. Branca, F. De Martini, L. Hardy, and S. Popescu, Phys. Rev. Lett. 80, 1121 (1998).

[8] M. Żukowski, A. Zeilinger, M. A. Horne, and A. K. Ekert, Phys. Rev. Lett. 71, 4287 (1993).

[9] S. Bose, V. Vedral, and P. L. Knight, Phys. Rev. A 57, 822 (1998).

[10] J-W. Pan, D. Bouwmeester, H. Weinfurter, and A. Zeilinger, Phys. Rev. Lett. 80, 3891 (1998).

[11] G. Krenn and A. Zeilinger, in Fundamental Problems in Quantum Theory: A Conference Held in Honor of Professor John A. Wheeler, edited by D. M. Greenberger and A. Zeilinger [Ann. N. Y. Acad. Sci. 755, 873 (1995)]; 
Phys. Rev. A 54, 1793 (1996); ibid 55, 3970 (1997); ibid 56, 4336 (1997).

[12] L. Vaidman, Los Alamos e-print archive, quant$\mathrm{ph} / 9810089$.

[13] N. D. Mermin, Pramana (to be published). See also Los Alamos e-print archive, quant-ph/9609013.

[14] N. D. Mermin, Am. J. Phys. 66, 753 (1998). See also Los Alamos e-print archive, quant-ph/9801057.

[15] N. D. Mermin, Found. Phys. (to be published). See also Los Alamos e-print archive, quant-ph/9807055.

[16] A. Cabello, Phys. Rev. A 59 (1999).

[17] D. M. Greenberger, M. A. Horne, and A. Zeilinger, in Bell's Theorem, Quantum Theory, and Conceptions of the Universe, edited by M. Kafatos (Kluwer, Dordrecht, 1989), p. 69.

[18] N. D. Mermin, Phys. Today 43 (6), 9 (1990); Am. J. Phys. 58, 731 (1990).

[19] D. M. Greenberger, M. A. Horne, A. Shimony, and A. Zeilinger, Am. J. Phys. 58, 1131 (1990).

[20] S. Kochen and E. P. Specker, J. Math. Mech. 17, 59 (1967).

[21] N. D. Mermin, Phys. Rev. Lett. 65, 3373 (1990); Rev. Mod. Phys. 65, 803 (1993).

[22] L. Hardy, Phys. Rev. Lett. 68, 2981 (1992).

[23] S. L. Braunstein, A. Mann, and M. Revzen, Phys. Rev. Lett. 68, 3259 (1992).

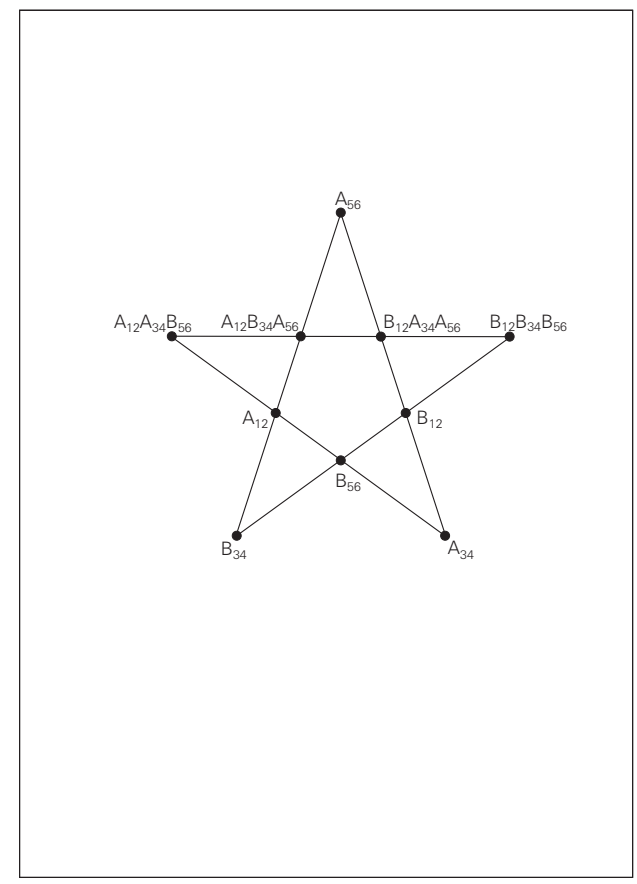

FIG. 1: Each dot represents an observable. The ten observables provide a proof of the Kochen-Specker theorem in a Hilbert space of dimension sixty four. The four observables on each line are mutually compatible and the product of their results must be positive, except for the horizontal line, where the product must be negative. 